\title{
Strengthening links between health action zone evaluation and primary care research
}

Dara Coppel Nottingham Health Action Zone Evaluation Manager, Nottingham City Primary Care Trust, Nottingham, UK and Dr Jane Dyas Trent Focus Local Co-ordinator, Division of General Practice, University of Nottingham, Nottingham, UK

There is an emphasis on developing partnerships throughout the Department of Health and other Government departments. Health Action Zones (HAZs) highlight the need for flexible working across boundaries at a local level. How to evaluate these partnerships in a manner that is practicable, pragmatic, straightforward for researchers and non-researchers alike is an issue that needs to be addressed. This paper assesses the potential of a community based evaluation tool - the Theories of Change Model - as an approach to evaluating partnerships. The model was applied to a very focused partnership between the Nottingham HAZ Evaluation Manager and the Local Co-ordinator of Trent Focus (an organisation funded by the NHSE Trent, to promote research and development in primary care). The aim was to provide an opportunity to portray the usefulness and limitations of implementing a theory based approach to 'valuing' primary care partnership working at a local level. The evaluation process itself was an important element of the formulation and implementation of the partnership. It helped to decide the aims and objectives of the partnership and proposed a theory as to why the partnership was worth investing in. At a minimum, if applied at the inception of a new partnership, the model was seen as a very useful planning tool. The process was very time consuming and to be most effective, needed the commitment from all key stakeholders involved in the partnership. This paper is an attempt to provide researchers/evaluators, in the widest sense, with a possible framework that would allow organizations to acknowledge, justify and address complex partnership working for health gain. This paper is of importance to anyone interested in defining and measuring desired processes and outcomes from partnership working.

Key words: evaluation; partnerships; primary care research

\section{Introduction}

Health inequalities is the most commonly used term in Europe to indicate the virtually universal phenomenon of variation of health by socio-economic status. In other words, poorer people have poorer health. There is compelling evidence that people who live in disadvantaged circumstances suffer from more illness, greater distress and have shorter lives than those who are more affluent

Address for correspondence: Dara Coppel, Health Action Zone Evaluation Manager, Nottingham Health Authority, 1 Standard Court, Park Row, Nottingham, NG1 6GN, UK. Email: dara. coppel@nottinghm-ha.trent.nhs.uk
(Whitehead and Diderichson, 1997; Wilkinson, 1997). In England, more attention has been paid to this since the publication of the Black Report which explained the extent of and trends in inequalities in health (Townsend, Davidson and Whitehead, 1988). In response, there are now a plethora of modernization programmes in Britain, set up through various government departments to tackle health inequalities.

Central to tackling health inequalities and the government's modernization agenda is partnership working. All the 'new' NHS White Papers recommendations on public health hinge on joined up working' (Secretary of State for Health, 1997; 1998; 1999). The NHS Plan, published in July 
2000, took this commitment further, stating that the NHS cannot tackle health inequalities alone (Secretary of State for Health, 2000). More specifically, it established the need for 'partnerships and co-operation at all levels of care between patients, carers and families and NHS Staff; between the health and social care sector; between different Government departments; between the public sector, voluntary organisations and private providers in the provision of NHS service - to ensure a patient-centred service.' (Secretary of State for Health, 2000, p 5). Thus, at a local level, a range of policy initiatives - Health Improvement Plans (HImPs), Primary Care Organisations (PCO's), Local Strategic Partnerships (LSPs) created through the New Commitment to Neighbourhood Renewal and Health Action Zones (HAZs) have all given impetus to tackling local needs through a partnership approach. They seek to unite all the relevant organisations operating on the ground to improve the well-being of communities across the country (Local Government Association, 2000).

In 1997, the announcement of the Health Action Zone initiative in Britain (National Health Service Executive, 1997) was but one programme in place to make an effective local contribution to reducing inequalities and modernizing services. Since 1998, twenty-six deprived areas in England were successfully given HAZ status. From the outset, partnership working and empowerment of deprived local communities was key to HAZs being able to achieve their aims (National Health Service Executive, 1997). Amery (2000) observed the differing partnership complexities within and across HAZs yet confirmed the importance of underpinning inter-professional collaboration in the Health Action Zones no matter what point they are starting at.

There is no doubt that true partnership working is difficult to achieve and although partnership working is a must, there are, at present, no government monitoring or performance management targets to measure it (Maddock, 2000). The need to evaluate joint working between HAZs and primary care has been recognised by the National HAZ Support Network (Billingham and Drinkwater, 2001). This paper aims to discuss the application of a community based evaluation tool - the Theories of Change - to a partnership across primary care and a Health Action Zone. By doing this, it hopes to portray the usefulness and limitations of implementing a theory based approach to 'valuing' partnership working. This paper is of importance to any researcher/evaluator interested in defining and measuring desired outcomes from partnership working and identifying good practice in primary care and other settings. It will offer key learning for all area based initiatives and other modernization programmes that need an insight into planning and evaluating effective partnerships.

\section{Background}

\section{The partnership}

The importance of partnerships in primary health care and primary care is not new (World Health Organisation, 1978). The partnership being evaluated in this paper is that between a coordinator of a primary care research network (Trent Focus) and the HAZ Evaluation Manager for the Health Action Zone in Nottingham. Both posts were representing the interface between their respective organisations. The aim of the partnership was to support the evaluation across the HAZ to ultimately provide an evidence base for the innovative community services/projects underway within the HAZ programme and to increase the capacity of the primary care research community.

As part of the overall evaluation strategy to prove partnership working within HAZ, the representative from Trent Focus and the Nottingham HAZ Evaluation Manager agreed to evaluate the specific working relationships that constituted their partnership. From the Trent Focus perspective the investment of time in networking, collaborating and integrating warranted an evaluation of the partnership. It also provided a unique opportunity to evaluate an aspect of Trent Focus activity not currently assessed.

\section{The theories of change model}

'A theory is a set of interrelated concepts, definitions and propositions that present a systematic view of events and situations by specifying relations among variables, in order to explain and predict the events or situations' (National Cancer Institute, 1995, p 6). Evaluators recognize the value of theory and as a result numerous theories and theoretical frameworks exist (National Cancer Institute, 1995). The 'Theory of Change' approach 
to evaluation is one theoretical framework, originally developed to find ways of evaluating community based programmes. Members of the Aspen Institutes Roundtable on Comprehensive Community Initiatives for children and families have been, for several years, exploring the theories of change approach as an alternative evaluation strategy to the traditional, scientific experimental design (Connell et al., 1995). It is a framework that is currently being tried and tested to share the pursuit of answers to WHY? WHAT? and HOW? interventions work (Jacobs, 2001; Judge et al., 1999; Milligan et al., 1999). Similar to the Realistic Evaluation framework by Pawson and Tilley (1997), the Theories of Change approach is defined as a 'systematic and cumulative study of the links between activities, outcomes and context of the initiative' (Connell and Kubisch, 1996). According to Weiss (1998), the approach should make explicit a sequence of assumptions/links that show how the project inputs (such as staff, resources and activities) translate through a series of intermediate steps to the desired project outcomes (such as improvements in peoples health, organizational change or the development of healthy communities). It is a set of hypotheses upon which people build their project plans. Simply, it is a set of beliefs that underlie action (Weiss, 1998). The microsteps of the theory/beliefs can then become the framework for the evaluation study which will determine if and how the linkages occur. The backbone to the framework process is being able to articulate and get consensus amongst all key stakeholders about the theory of change in the early stages of the initiative (Judge et al, 1999).

For the purposes of this evaluation the approach was applied to a partnership rather than to a community based initiative. To our knowledge, this is the first example of using the Theories of Change approach to purely focus on the investigation of effective partnership working.

\section{Method}

The theories of change evaluation model was being used by the national HAZ evaluation team to evaluate all twenty six HAZs across England (Judge et al., 1999) and was recommended for use in local HAZ evaluations. The Nottingham HAZ
Evaluation Manager consequently developed a resource pack for use with local project leads and stakeholders to evaluate their own initiatives (Coppel, 1999). The resource pack was piloted in the evaluation of this partnership. The application of the evaluation framework (Theories of Change) to the partnership was guided by the questions (steps) highlighted in the locally devised resource pack (see Table 1).

The implementation of the model occurred over three separate face to face meetings between the two partners. It was beyond the scope of this exercise to collect the data by which to assess the success or otherwise of the partnership as would be done with a full evaluation. Neither did we return to the framework to assess the additional consequences and outcomes that might be expected to evolve from a successful partnership in the course of its development and maturation.

\section{Findings}

Figure 1 illustrates the information captured through the implementation of the theories of change evalu-

Table 1 Questions (steps) used to help elicit a theory of change for partnership working between Trent Focus And Nottingham health action zone

\section{QUESTION 1}

What is the context in which you are working that has made you see the value of setting up this project?

QUESTION 2

What activities are you doing or planning to do within the project?

QUESTION 3

What initial results do you expect from the project? QUESTION 4

What medium term outcomes do you expect from the project?

QUESTION 5

What long-term outcomes do you expect from the project?

QUESTION 6

How will the initial results lead to the medium term outcomes?

QUESTION 7

How do the medium term outcomes lead to the long-term outcomes?

QUESTION 8

What barriers do you foresee in implementing the actions or activities? 

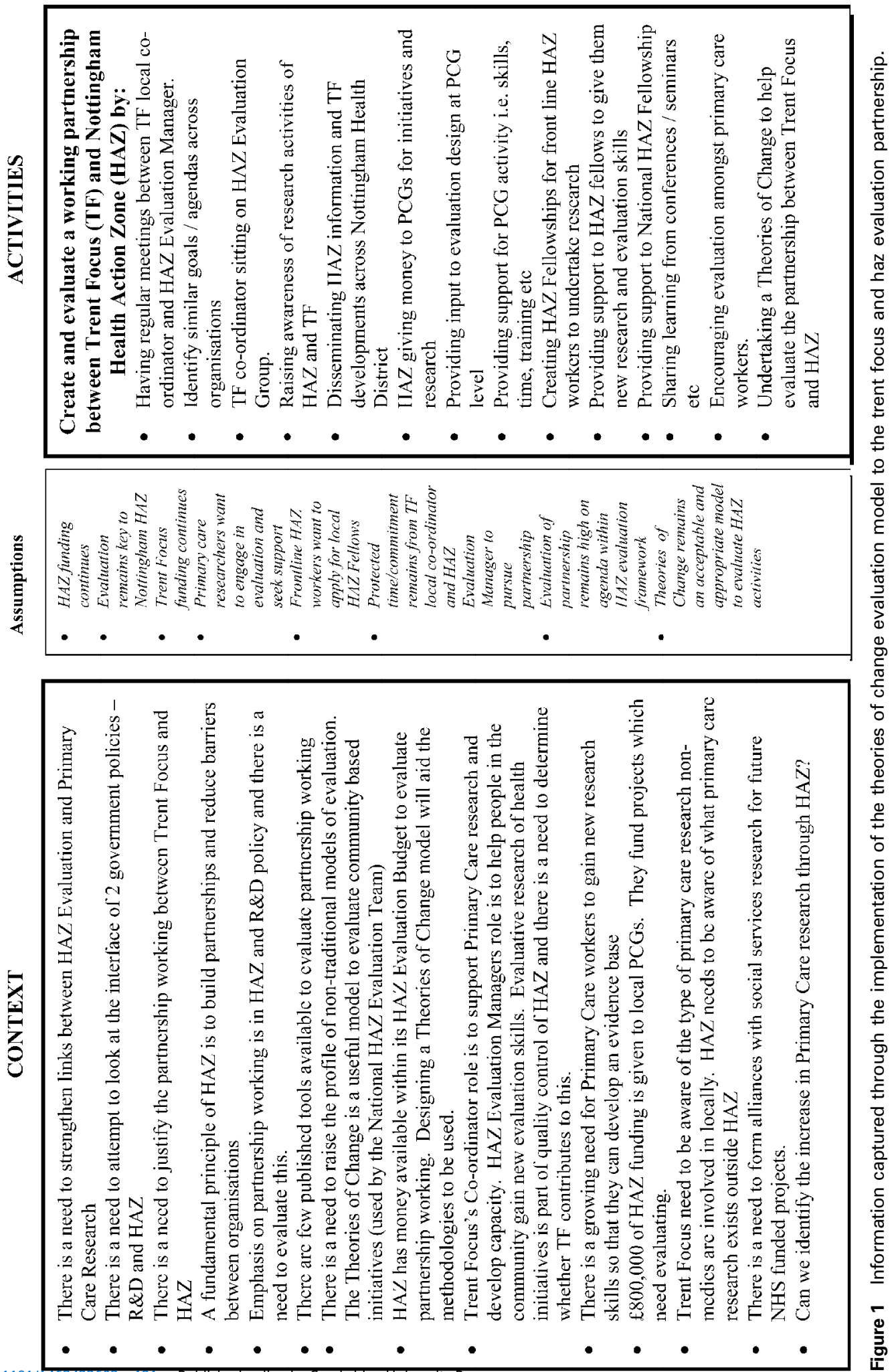

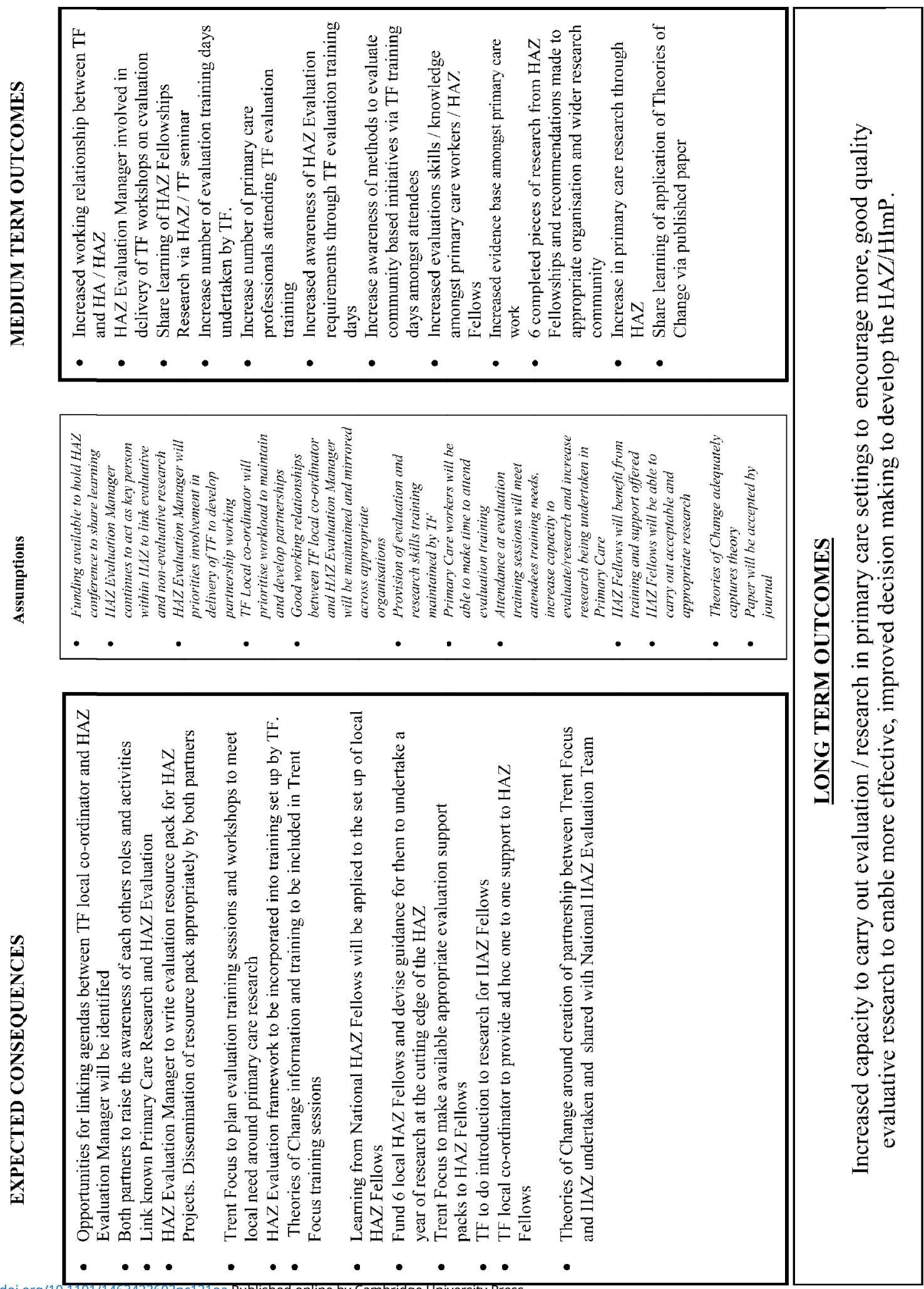

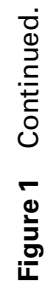


ation model to the Trent Focus and HAZ Evaluation partnership.

\section{Discussion}

The application of the Theories of Change evaluation model developed here and presented in Figure 1 utilized a process-orientated approach to the evaluation of partnership working. Within this framework the evaluation process is usually considered as a continuous activity. However, since the purpose of this paper was to provoke discussion about the usefulness and limitations of the tool itself in valuing partnership working, our comments are focussed on information captured at just one point in the evolution of the partnership.

From the outset, there was a drive for both organizations to collaborate because of the belief that the same ends could be achieved more efficiently by working together rather than separately and that joint working should assume joint evaluation (Amery, 2000). A significant investment in time, energy and commitment by the two partners in applying the Theories of Change enabled this process. By working through the series of questions within the resource pack (Table 1), key aims and goals of the joint working were discussed and agreed, as well as the means to achieving these goals and the intermediate outcomes that should be achieved on the way to reaching stated goals. For example, it became clear that one of the fundamental aims of Trent Focus and the HAZ Evaluation was to build up research/evaluation capacity amongst front line workers. This was not the reason why the partners initially chose to work together yet its identification has since led to a multitude of activities and events that have captured the opportunities and maximized the chances of empowering professionals and communities with evaluative research skills. The evaluation was viewed as a natural extension of the collaborative process. Both partners welcomed the opportunity to work together to specify the assumptions and theories that guided decisions about the structure and components of the partnership. Early involvement in the evaluation process built trust amongst both partners and their organizations.

\section{Weakness of the approach}

One of the most challenging aspects of the Theories of Change is the gaining of consensus among the many stakeholders involved in implementing the partnership working (Judge, 2000). Previous authors and ourselves have recognized that the involvement with as many key stakeholders within the process itself would inevitably enrich both the activities and the lessons to be learnt from it as well as increasing evaluation skills (Weiss, 1995: Judge, 2000). In an ideal world, there would have been an attempt to capture as many stakeholders views and opinions as possible. In this particular partnership, the stakeholders were identified as the evaluation and research community with whom we work and other key members of our organizations. Unfortunately it was not feasible to carry out such a resource intensive exercise. Nonetheless both partners felt that the added benefit of using the Theories of Change model for evaluating the partnership justified the articulation of a 'theory' without the direct involvement of other stakeholders.

\section{Usefulness and limitations of using the Theories of Change Model to value partnership working}

\section{Planning}

Weiss and colleagues (1995) believe that the Theory of Change can sharpen the planning and implementation of an initiative. Without a doubt the planning was the most valuable aspect of the application of the Theories of Change to the partnership. In some respects, the complexity of the theories could make it difficult to see meaningful evaluation opportunities. However, without a clear theory of change mapped out, activity and outcomes would have been ad hoc, immeasurable and potentially destined to fail. It was felt that the approach made plans more sensible and more grounded in current research, making successful implementation more likely. At the very least, the bringing together of the two partners to articulate the contexts, mechanisms and outcomes and underlying theory actually enhanced the depth of partnership working. It also justified the input made by both partners into the partnership by allowing progress to be assessed along the way and making us accountable as people and organizations for fulfilling our commitments. 


\section{Communication and Terminology}

Another factor that we have identified as contributing to the successful application of the theories of change in evaluating a partnership focuses on aspects of communication. Defining the terminology for any evaluation is of extreme importance. Connell and Kubisch (1998) state that when applying the Theories of Change approach to evaluation, the language used to establish the theory impacts upon the final decisions. The resource pack that was devised before the implementation of the Theories of Change provided an agreement on the terminology. This undoubtedly avoided time being spent on understanding each and every term used by either partner (Coppel, 1999).

\section{Theoretical Pathways}

It is impossible to use traditional evaluation methods, such as randomized controlled trials to evaluate the social phenomena of the partnership. However, by using the Theories of Change evaluation model we were able to make explicit the important pathways of change that were expected. This proved to be useful for providing feedback that distinguished failure of the theory generated, as separate from failures in the actual implementation of the partnership working. This may be significant for partnerships to enable them to continue working together. The generation of theory also facilitated the identification of the appropriate measurement and data collection methods needed to prove the success of the partnership. This is in agreement with findings of Connell and Klem (1999) and Judge (2000). Although it was easy to make explicit the detailed steps (theory) along the pathway of change, it was difficult to find a point at which to stop. We generated a considerable amount of theory and so many detailed activities for which data could potentially be collected. This raises important issues for the ensuing evaluation. As with any evaluation a prioritization process has to take place to identify the important criteria by which to assess the value and success or otherwise of the partnership. In other words, given that time and finances is limited to maintain regular meetings between stakeholders and also for data collection/analysis, it is neither possible nor desirable to evaluate everything.

The potential to generate huge amounts of data and the additional pressures on key stakeholders, as identified in this study, mirrors the findings of others who have used the Theories of Change model (Weiss, 1998). The implication of this for evaluating the increasing number of multipartnerships in primary care, is that greater prioritization will be required to determine which steps in the sequence of events merits data collection and analysis.

From our experience it would be unfair to say that the application of the Theories of Change to partnership working was easy. A Theory of Change approach is a complex undertaking (Milligan et al., 1999). It requires a devoted investment in time to be able to develop a model, which is plausible, achievable and testable. A shared understanding of the Theories of Change and its application was fundamental to us proceeding with this project. This task was certainly made easier by the articulation and implementation of the steps outlined in the resource pack (Coppel, 1999). To date few authors have attempted to define steps to carry out a Theory of Change, and little is currently known about the processes evaluators should follow in carrying them out (Milligan et al., 1999). The process we used, successfully elicited an in-depth overview of partnership working allowing it all to be captured on three pages. This provided an easy document to distribute to stakeholders. It was short, concise and specific enough to reach a wide audience. Having an accepted and effective model was also seen as a positive short-term output. The document needs to be seen as a working document with the need to revisit the assumptions on a regular basis. This is not only time consuming but may be a vulnerable activity in times of rapid contextual change. The reorganization of the Health Authorities and the NHS Executive offices (Department of Health, 2001) as well as changes in the structure of funding for both HAZ and NHS R\&D (Department of Health, 2000), will have a direct impact on this partnership. The influence of external factors will exist for all partnerships and similarly will have an effect on any evaluation approach. From our experience we can only suggest that the very emphasis on identifying the underlying assumptions of the partnership, as occurs with the Theories of Change, can help a partnership to plan and predict future directions in changing climates. 


\section{Conclusion}

The evaluation framework presented here is offered as a contribution to understanding the process of implementing a learning tool for those engaged in partnership working. It is important to make it clear that the Theories of Change evaluation framework is one approach to evaluating community based programmes or activities. However, in our experience, articulating a theory of change through collaborative process has its difficulties yet we deemed it to be a very useful exercise. It certainly attempts to throw light on the effectiveness of partnership working. It is most powerful in attempting to determine not just how the partnership needs to be working but why it should work. Effective use of the theory of change could need practice, but both partners in this study considered that the pay off was well worthwhile.

Testing the framework by carrying out a full evaluation should form the basis of future research. We also recommend that further work needs to be done to evaluate and critically appraise the Theories of Change model against other published tools for assessing partnership working.

The commitment and energy to evaluate partnership working remains important to ensure that we are not perpetuating ineffective partnerships and that valued partnerships are sustainable. This is both relevant and timely given the greater emphasis on partnership working in primary care trusts and all health and social services fields.

\section{References}

Amery, J. 2000: Interprofessional working in Health Action Zones: how can this be fostered and sustained? Journal of Interprofessional Care Vol. 14, No 1.

Billingham, K. and Drinkwater, C. 2001: Working together, Changing Together? Health Action Zones and Primary Care. HAZ National Support Network, (Unpublished).

Connell, J. and Klem, A. 1999: You Can Get There From Here: Using a Theories of Change Approach to Plan Urban Education Reforms. Institute for Research and Reform in Education, (Unpublished).

Connell, J.P. and Kubisch, A.C. 1996: Applying a Theories of Change Approach to the Evaluation of comprehensive Community Initiatives. Washington, DC: The Aspen Institute.

Connell, J.P. and Kubisch, A.C. 1998: Applying a Theory of Change Approach to the Evaluation of Comprehensive Community Initiatives: Progress, Prospects, and Problems. In
Fulbright-Anderson, K., Kubisch, A.C. and Connell J.P editors, New Approaches to Evaluating Community Initiative. Volume 2 Theory, Measurement and Analysis, Washington DC: The Aspen Institute.

Connell, J.P., Kubisch, A.C., Schorr, L.B. and Weiss, C.H. editors 1995: New Approaches to Evaluating Community Initiatives: Concepts, methods and contexts. Washington DC: The Aspen Institute.

Coppel, D. 1999: An Evaluation Resource Pack for Nottingham HAZ Projects. Unpublished.

Department of Health 2000: Research and Development for a First Class Service R\&D - funding in the New NHS. London: Department of Health.

Department of Health 2001: Shifting the Balance of Power within the NHS Securing Delivery. London: Department of Health.

Jacobs, B. 2001: Evaluating a Complex Partnership: North Staffordshire Health Action Zone. Staffordshire University.

Judge, K., Bauld, C., Adams, C., Findlater, J., Killoran, A., Barnes, M. and Benzeval, M. 1999: Health Action Zones: Learning to make a difference. University of Kent at Canterbury: PSSRU.

Judge, K. 2000: Testing Evaluation to the Limits: the case of English Health Action Zones. Journal of Health Services Research Policy 5, pp 3-5.

Local Government Association 2000: Supporting the Action Zones - early messages, future plans. London: LGA Publications.

Maddock, S. 2000: Managing the Development of Partnerships in Health Action Zones. International Journal of Health Care Quality Assurance 13, 65-73.

Milligan, S., Coulton, C., York P. and Register, R. 1999: Implementing a Theory of Change Evaluation in the Cleveland Community-Building Initiative: A Case Study. In: Connell, J. P., Kubisch, A.C., Schorr, L.B., and Weiss C.H. editors, New Approaches to Evaluating Community Initiatives: Concepts, methods and contexts. Washington DC: The Aspen Institute.

National Cancer Institute 1995: Theory at a Glance: A Guide for Health Promotion Practice (NIH Publication No. 97-3896). Bethesda, MD: National Cancer Institute.

National Health Service Executive (EL(97)65). 1997: Health Action Zones - Invitation to Bid. NHSE Department of Health.

Pawson, R. and Tilley, N. 1997: Realistic Evaluation. London: Sage Publications.

Secretary of State for Health 1997: The New NHS, Modern, Dependable. London: Stationary Office.

Secretary of State for Health 1998: Our Healthier Nation. London: Stationary Office.

Secretary of State for Health 1999: Saving Lives: Our Healthier Nation. London: Stationary Office.

Secretary of State for Health 2000: The NHS Plan. Norwich: HMSO.

Townsend, P., Davidson, N. and Whitehead, M. 1988: Inequalities in health: the Black Report and the Health Divide. 2nd edition. London: Penguin.

Weiss, C.H 1995: Nothing as practical as good theory: exploring theory-based evaluation for comprehensive community initiat- 
ives for children and families, In: Connell, J.P., Kubisch, A. C., Schorr, L.B. and Weiss, C.H. editors, New Approaches to Evaluating Community Initiatives: Concepts, methods and contexts. Washington DC: The Aspen Institute.

Weiss, C.H. 1998: Evaluation. 2nd Edition. London: Prentice Hall. Whitehead, M. and Diderichsen, F. 1997: International evidence on social inequalities in health. In: Drever, F., Whitehead M. editors, Health inequalities: decennial supplement: DS Series no.15. London: The Stationery Office.

Wilkinson, R.G. 1997: Health Inequalities: relative or absolute material standards? British Medical Journal 314, 591-95.

World Health Organisation 1978: The Alma Ata Declaration. Geneva: World Health Organisation. 